\title{
Evaluación de extractos de ocho especies vegetales en el control de mildeo velloso (Peronospora destructor Berk) en cebolla de bulbo (Allium cepa L.).
}

\section{Evaluation of eight extracts of plants in the control of mildeo velloso (Peronospora destructor Berk) in onion bulbs (Allium cepa L.).}

Pedro Enrique Cubides Hernández ${ }^{1}$

\begin{abstract}
Resumen
Extractos de ocho especies vegetales: eucalipto (Eucalytus globulus), cola de caballo (Equisetum bogotense), ortiga (Urtica urens), manzanilla (Matricaria chamomilla), caléndula (Calendula officinalis), yerbabuena (Menta viridis), ajo (Allium sativum) y clavo (Syzygum aromaticum), preparados por el método de decocción $(100 \mathrm{~g} / \mathrm{l})$, fueron evaluados en cuanto a su eficiencia en el control de $P$. destructor en cebolla de bulbo. Se utilizó el diseño experimental de bloques al azar con cuatro repeticiones y siete aplicaciones $(52,60$, 68, 76,84, 92 y 100 días después del trasplante), incluyendo un testigo químico Ridomil (metalaxil + mancozeb) (2 g/l) y el testigo absoluto; se evaluaron la incidencia y severidad de $P$. destructor, y el número y el peso de bulbos cosechados. El ANOVA mostró que de las siete aplicaciones realizadas, la última presentó efectos estadísticos en el control de la incidencia y severidad de $P$. destructor. El extracto de manzanilla superó al Ridomil en el control de la incidencia y severidad de $P$. destructor sin diferencias estadísticas entre sí, y fue significativamente mejor al observado con los extractos de eucalipto, cola de caballo, caléndula y el testigo; el extracto de manzanilla
\end{abstract}

\begin{abstract}
The extracts from eight plant species: eucalyptus (globulus Eucalytus), ponytail (Equisetum bogotense), nettle (Urtica urens), camomile (Matricaria chamomilla), marigold (Calendula officinalis), Yerbabuena (Mentha viridis), garlic (Allium sativum) and clove (Syzygum aromaticum), prepared by the method of decoction (100 g / I) were evaluated for their efficiency in controlling $P$. destructor on bulb. Was used an experimental design a randomized block with four replications and seven applications $(52,60,68,76,84,92$ y 100 days after transplant) including a chemical witness Ridomil (metalaxyl + mancozeb) $(2 \mathrm{~g} / \mathrm{I})$ and the absolute witness, were evaluated the incidence and severity of $P$. destructor, the number and weight of bulbs harvested. The ANOVA showed that seven of the applications made, the last one showed statistical effects in the control of incidence and severity of $P$. destructor. Chamomile extract to exceed to Ridomil controlling the incidence and severity of $P$. destructor without statistical differences each other and was significantly better than extracts of eucalyptus, ponytail, marigold and the witness; chamomile extract surpass the chemical witness in the number and weight of
\end{abstract}

Ingeniero Agrónomo. Universidad Pedagógica y Tecnológica de Colombia. e-mail: pedro.cubides@uptc.edu.co 
superó al testigo químico en el número y peso de bulbos de cebolla sin presentar diferencias estadísticas, y fue significativamente mejor al obtenido con los extractos de eucalipto, cola de caballo, ortiga, caléndula, yerbabuena, clavo y el testigo absoluto. El extracto de manzanilla es una alternativa ecológica que se debe tener en cuenta en el manejo integrado de $P$. destructor en cebolla de bulbo.

Palabras clave: Extractos vegetales, Peronospora destructor, Cebolla. (Fuente: USDA) onion bulbs without statistical differences and it was significantly better than extracts of eucalyptus, ponytail, nettle, calendula, yerbabuena, clove and the absolute witness. The extract of chamomile is an ecofriendly alternative to be taken into account in the integrated management of $P$. destructor in onion bulb.

Key words: Plant extracts, Peronospora destructor, Onion. (Source: USDA) 


\section{Introducción}

El área sembrada de cebolla de bulbo en Colombia es de 12.126 ha, para una producción de 262.430 toneladas (t); aproximadamente el $40 \%$ de esta área se encuentra en el departamento de Boyacá, para una producción de 104.793 t, lo que evidencia su gran importancia socio-económica para el departamento (Ministerio de Agricultura, 2005). El $85 \%$ de la agricultura de la provincia del Tundama corresponde al cultivo de la cebolla; allí el monocultivo está generando que los rendimientos por unidad de área se vayan reduciendo, debido a los problemas fitosanitarios, al igual que al mal uso y manejo de los agroquímicos (Albeiro, 1989; Aponte y Alfonso, 2008).

Una de las enfermedades que más afectan la producción y la calidad de los bulbos de la cebolla es el mildeo velloso ( $P$. destructor), que se encuentra ampliamente distribuido por todo el mundo (Agrios, 1996; Brewster, 2001), y causa grandes pérdidas económicas, que se traducen en detrimento del nivel de vida de los cultivadores de la región.

El control del mildeo velloso es químico, y la aplicación masiva de fungicidas puede estar ocasionando la aparición de cepas del hongo con resistencia a éstos, y para controlarlo se deben aumentar las frecuencias de aplicación o las dosis; debido a esto, se plantea la necesidad de evaluar otras alternativas de control, como son los extractos vegetales, con el propósito de reducir pérdidas y aumentar los ingresos de las familias que viven del cultivo de la cebolla de bulbo.

\section{Materiales y Métodos}

La evaluación de los extractos de ocho especies de plantas en el control de P. destructor se realizó en la granja Surbatá del ICA, en el municipio de Duitama, localizada a 2535 m.s.n.m., en la latitud $-0,73.07$ y longitud 05,79 (Alcaldía de Duitama, 2012). En la preparación del lote se hizo un pase de arado a $30 \mathrm{~cm}$ de profundidad y dos pases de rastrillo, uno perpendicular al otro. El lote se dividió en cuatro bloques, cada uno de $3 \mathrm{~m}$ de ancho y $60 \mathrm{~m}$ de largo; por bloque se trazaron 10 parcelas de $5 \times 2 \mathrm{~m}$; entre parcelas y bloques se dejó $1 \mathrm{~m}$ de distancia, para un total de área de 793 $\mathrm{m}^{2}$. Para el transplante se obtuvieron las plántulas del híbrido cebolla de bulbo yellow granex, de un semillero de 65 días, ubicado en la vereda Punta Larga, Nobsa, Boyacá. Se hizo dejando 0,10 m entre plantas (51 plantas/surco) y $0,20 \mathrm{~m}$ entre surcos (11 surcos/parcela), para un total de 561 plantas por unidad experimental.

Para la preparación de los extractos de cada una de las ocho especies de plantas medicinales se cortó el material seleccionado y se tomaron $100 \mathrm{~g}$ de tejido fresco, los cuales se dejaron 24 horas en un recipiente con 1 litro de agua; al cabo de este tiempo se pusieron a hervir a fuego lento durante 15 minutos, contados a partir del primer hervor; se dejó enfriar, se filtró y se aforó.

Las aplicaciones se hicieron con una bomba de espalda, y se iniciaron con la aparición de los primeros síntomas de la enfermedad; la frecuencia fue semanal, durante siete semanas; se incluyeron dos testigos: el químico (Metalaxil+Mancozeb [Ridomil]), con el mismo número de aplicaciones semanales, y el testigo absoluto, sin aplicaciones. La fertilización se hizo en dos aplicaciones: la primera a los 15 días, y la segunda a los 2 meses después del trasplante, y el riego se realizó durante todo el ciclo del cultivo, teniendo en cuenta el clima.

Se utilizó un diseño experimental de bloques al azar, con 8 tratamientos y 4 repeticiones, de los cuales hubo un testigo químico (Ridomil) y el testigo absoluto. Se evaluó la incidencia, que se estableció contando cada ocho días el número de plantas que presentaban los síntomas de la enfermedad en los cinco surcos centrales de cada una de las parcelas; el conteo se realizó cada semana durante el ciclo del cultivo. En la severidad, el porcentaje de tejido foliar se evaluó con la escala de Horsfall y Barrat, tomando datos semanales de las plantas de los cinco surcos centrales de cada unidad experimental. El número y el peso de bulbos se realizó en cosecha, teniendo en cuenta los diámetros de éstos. A los datos obtenidos se les aplicaron las pruebas estadísticas de ANOVA y Duncan.

\section{Resultados y Discusión}

Incidencia. El análisis del ANOVA mostró que los extractos no tuvieron efectos estadísticos en el control de la incidencia de $P$. destructor durante las seis primeras aplicaciones, y sí en la última, realizada a los 100 días después del trasplante.

El mejor control de la incidencia de $P$. destructor se dio con el extracto de manzanilla, con diferencias estadísticas significativas del 5\%, por la prueba de 
Duncan, con respecto al extracto de caléndula y al testigo absoluto; este resultado es importante porque muestra que el extracto de manzanilla contiene metabolitos con propiedades fungicidas o fungistáticas contra $P$. destructor, lo cual concuerda con el trabajo de Rojas (2001), quien también reportó la eficiencia de este extracto en el control de la pudrición del bulbo Sclerotium cepivorum; el extracto de manzanilla también ha sido reportado por su eficiencia en el control de otros fitopatógenos en otros cultivos, como en el caso de Colletotrichum en arveja (Espinel y Neira, 1998). El extracto de yerbabuena también mostró un efecto similar al testigo químico en el control de la incidencia y severidad de $P$. destructor.

Con el extracto de manzanilla la incidencia de la enfermedad fue menor a la registrada con los extractos de eucalipto, cola de caballo, ortiga, caléndula, ajo, yerbabuena, clavo y el testigo químico, sin diferencias estadísticas entre sí, lo cual muestra que la aplicación de los extractos vegetales tuvo un efecto similar al fungicida en el control de la incidencia de la enfermedad. Los extractos de manzanilla y de ortiga tuvieron un efecto similar en el control de la incidencia, y fueron mejores en comparación con el testigo, con diferencias significativas.

El extracto de eucalipto mostró mayor eficiencia en el control de la incidencia $P$. destructor durante las cuatro primeras aplicaciones, hechas a los 52 , 60,68 y 76 días después del trasplante, y un buen desempeño en el control durante el experimento. Con el extracto de ortiga, el mejor control de la incidencia de este fitopatógeno se dio con las aplicaciones realizadas desde los 68 hasta los 100 días; ocupando el segundo lugar de eficiencia en el control de la enfermedad, después de la manzanilla.

El testigo, durante todo el experimento, presentó mayor porcentaje de incidencia de $P$. destructor, acompañado del extracto de caléndula, que no mostró ningún efecto de control durante las cuatro últimas aplicaciones. El extracto de caléndula fue el menos eficiente en el control de la enfermedad; posiblemente, este extracto no contiene metabolitos con propiedades fungicidas, o el método de preparación utilizado no es el apropiado para la extracción de metabolitos. El extracto de caléndula también ha sido reportado por su deficiencia en el control de Monilinia fructigena en durazno Gayón.

En la figura 1 se muestra el comportamiento de los tratamientos en el control de la incidencia de $P$. destructor; se puede apreciar que el extracto de caléndula no influyó en el control de este fitopatógeno, presentando un comportamiento similar al testigo absoluto.

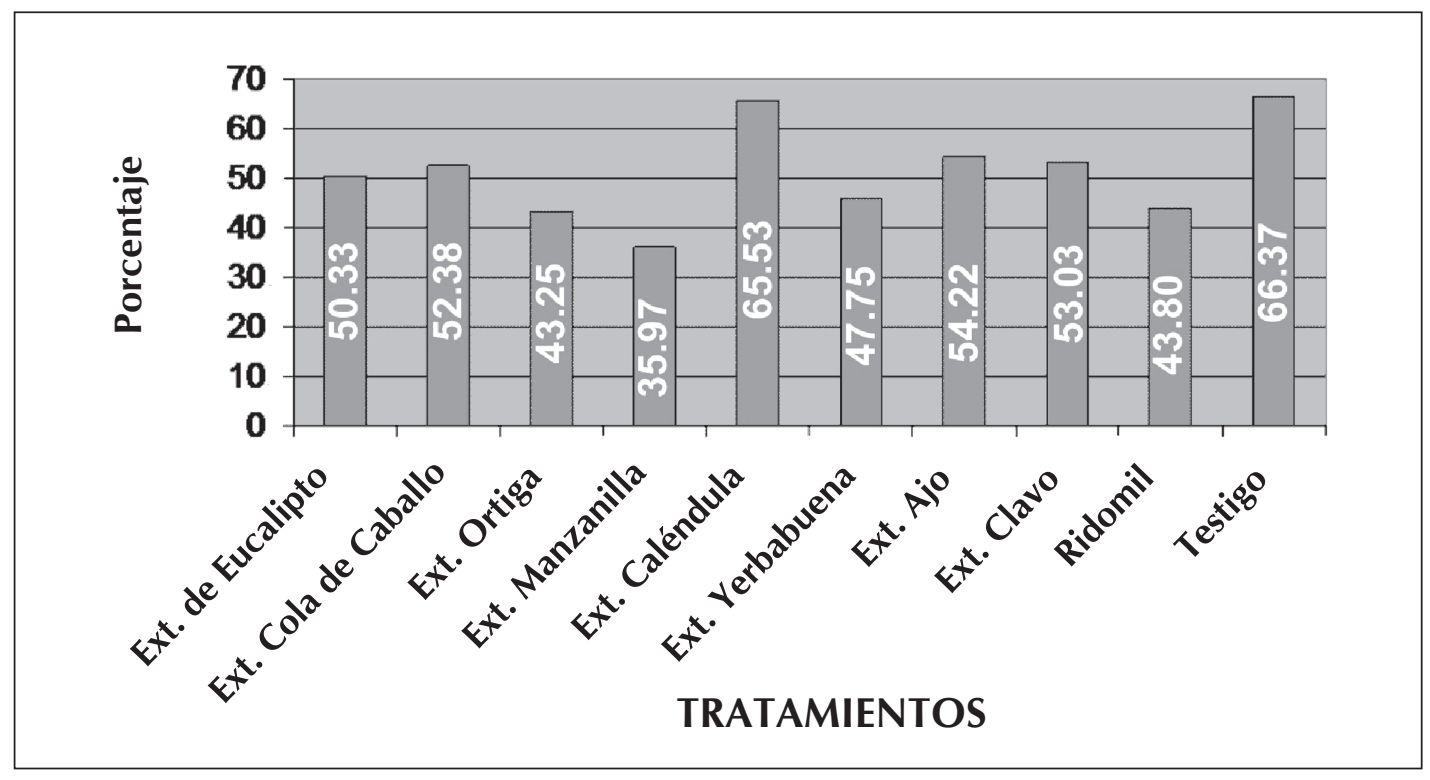

Figura 1. Efectos de los extractos vegetales en el control de la incidencia de $P$. destructor a los 100 días. 
Severidad. EI ANOVA mostró que la última aplicación de los tratamientos, realizada a los 100 días después del trasplante, tuvo efectos significativos en el control de la severidad de $P$. destructor. El testigo químico Ridomil presentó mayor control de la severidad de $P$. destructor con diferencias estadísticas significativas del 0,5\% por la prueba de Duncan, en comparación con el obtenido con los extractos de cola de caballo, de caléndula y de ortiga, y con el testigo.

El extracto de manzanilla superó a los extractos de cola de caballo, caléndula y al testigo absoluto en el control de la severidad de la enfermedad con diferencias estadísticas significativas del 0,5\% por la prueba de Duncan; durante las seis primeras aplicaciones, este extracto no tuvo diferencias estadísticas con el resto de los tratamientos, pero se observó un mejor control durante todo el experimento.

Los extractos de eucalipto, cola de caballo, ortiga, caléndula, ajo y clavo, con el testigo absoluto no presentaron diferencias estadísticas en el control de la enfermedad, lo cual muestra que el extracto de estas especies vegetales no afecta el desarrollo de la enfermedad.

El extracto de clavo, a pesar de tener menor efecto en el control de $P$. destructor, podría ser considerado una alternativa para el manejo de este fitopatógeno, pues ha mostrado eficacia en el control de otros fitopatógenos, como Colletotrichum sp. en curuba (Rodríguez y Velandia, 1996; Fonseca y Méndez, 1998); las propiedades fungicidas del clavo son atribuidas a su alto contenido de eugenol (García, 1992).

Con extracto de yerbabuena se presentó un leve control de la severidad de $P$. destructor durante las cuatro últimas aplicaciones (efectuadas de los 76 a los 100 días después del trasplante); con el extracto de ajo el ataque del hongo fue un poco menor que con el testigo absoluto durante las siete aplicaciones, y el extracto de cola de caballo fue el menos eficiente en el control de la severidad de la enfermedad durante todo el experimento (Tabla 2).

Tabla 1. Efecto de la aplicación de los extractos vegetales en el control de la severidad del $P$. destructor

\begin{tabular}{|c|c|c|c|c|c|c|c|}
\hline \multirow{2}{*}{ TRATAMIENTOS } & \multicolumn{7}{|c|}{ SEVERIDAD EN \% } \\
\hline & 52 días & 60 días & 68 días & 76 días & 84 días & 92 días & 100 días \\
\hline Ext. de Eucalipto & 0 & 7,5 & 15 & 40 & 56,25 & 68,75 & $81,25 \mathrm{abcd}$ \\
\hline Ext. Cola caballo & 0 & 7,5 & 17,5 & 37,5 & 60 & 73,75 & $87,50 \mathrm{a}$ \\
\hline Ext. Ortiga & 0 & 6,75 & 17,5 & 33,75 & 52,5 & 71,25 & $83,75 a b c$ \\
\hline Ext. Manzanilla & 0 & 7,5 & 20 & 31,25 & 48,75 & 61,5 & $73,75 \mathrm{~cd}$ \\
\hline Ext. Caléndula & 0 & 8,75 & 18,75 & 35 & 57,5 & 72,5 & $87,50 a$ \\
\hline Ext. Yerbabuena & 0 & 8,75 & 18 & 26,75 & 40 & 58,75 & $76,25 \mathrm{bcd}$ \\
\hline Ext. Ajo & 0 & 8,75 & 16,25 & 33,75 & 43,75 & 58,75 & $77,50 \mathrm{abcd}$ \\
\hline Ext. Clavo & 0 & 10 & 18,75 & 33,75 & 48,75 & 62,5 & $77,50 \mathrm{abcd}$ \\
\hline Testigo Químico & 0 & 11,25 & 22,5 & 33,75 & 42,5 & 58,75 & $72,50 \mathrm{~d}$ \\
\hline Testigo & 0 & 6,75 & 25 & 35 & 55 & 73,75 & $85,00 a b$ \\
\hline
\end{tabular}

Letras diferentes presentan diferencias estadísticamente significativas $(p<0.05)$

En la figura 2 se observa el comportamiento de los tratamientos en el control de la severidad de P. destructor; el mejor control con los extractos lo ejerció el de manzanilla, seguido del Ridomil. Con las aplicaciones del fungicida Ridomil se esperaba un control más eficiente de $P$. destructor, lo cual se dio posiblemente debido a cepas del hongo con tolerancia o resistencia a este fungicida, como ha sido reportado por Rubio y Tobar (2009), Castaño (1996) y Hard y Araya (1996). 


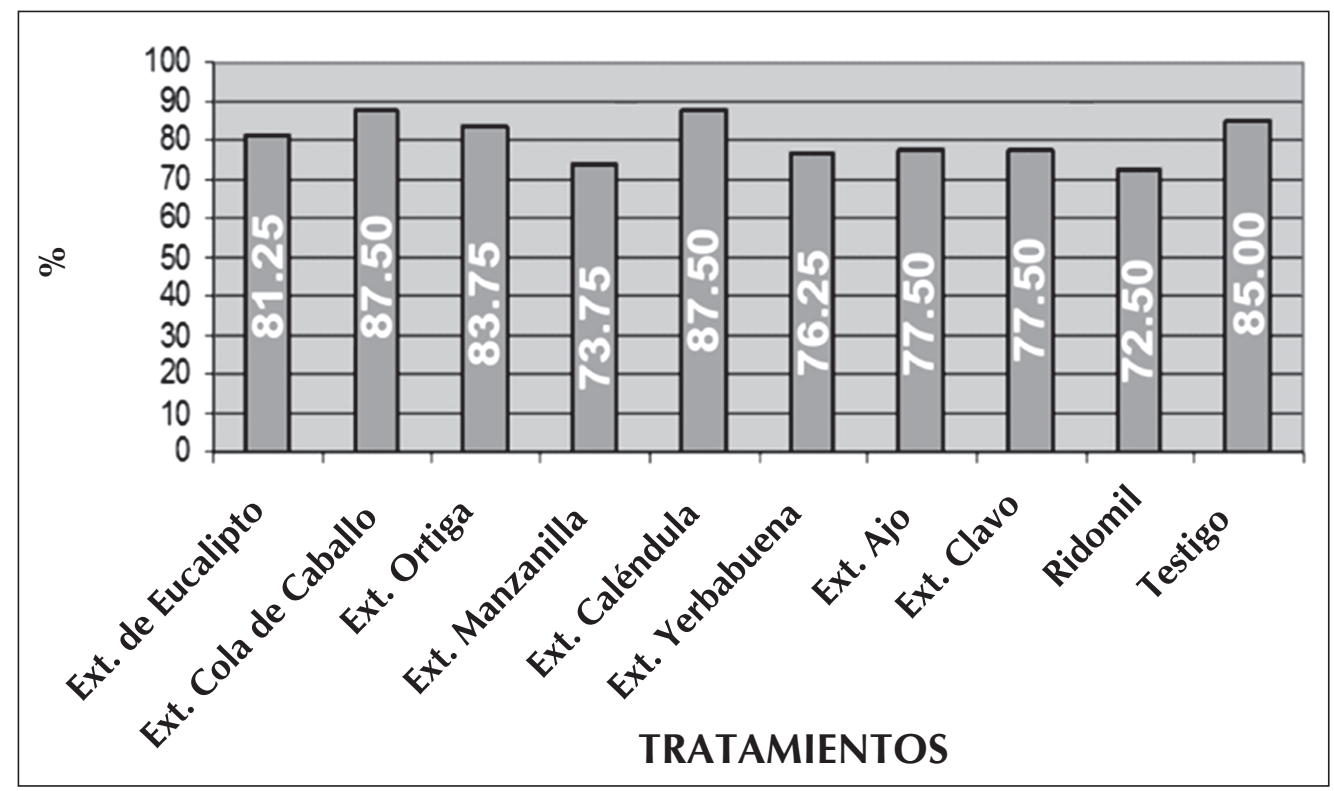

Figura 2. Control de la severidad con los extractos vegetales a los 100 días

Número de bulbos. El ANOVA mostró que con la aplicación de los extractos vegetales se obtuvieron diferencias estadísticas significativas en el número de bulbos de primera, y no lo fue en el número de bulbos de segunda y tercera.

El extracto de manzanilla obtuvo un promedio de 27 bulbos de primera, superando a los extractos de eucalipto, cola de caballo, ortiga, caléndula, yerbabuena y clavo, y al testigo absoluto con diferencias estadísticas significativas del $5 \%$ por la prueba de Duncan. El extracto de manzanilla superó al fungicida Ridomil (Metalaxil + Mancozeb) en el número y peso de bulbos de primera, $y$ fue significativamente mejor al obtenido con los extractos de eucalipto y cola de caballo, y con el testigo absoluto, lo que se explica por su mayor eficiencia en el control de P. destructor, posiblemente por el contenido del ingrediente activo borneol. El extracto de manzanilla superó al extracto de ajo y al tratamiento con el fungicida Ridomil en el número de bulbos de primera, sin diferencias estadísticas entre sí, lo cual es atribuido a su efecto en el control del ataque de $P$. destructor.

El número de bulbos de segunda fue mayor con el fungicida (Ridomil), superando ampliamente al extracto de eucalipto, con diferencias estadísticas del $0.5 \%$ por la prueba de Duncan.

Con los demás tratamientos, incluyendo a los testigos absoluto y químico, no se presentaron diferencias estadísticas significativas en relación con esta variable.

El número de bulbos cosechados de tercera fue mejor en el testigo absoluto, en comparación al obtenido con los demás tratamientos, con diferencias estadísticas del $5 \%$ por la prueba de Duncan, debido a un mayor ataque en la enfermedad.

Para el número total de bulbos cosechados no se presentaron diferencias estadísticas entre sí. 
Tabla 2. Número de bulbos cosechados según su tamaño

\begin{tabular}{|c|c|c|c|c|}
\hline \multirow{2}{*}{ TRATAMIENTOS } & \multicolumn{3}{|c|}{ NÚMERO DE BULBOS/TAMAÑOS } & \multirow{2}{*}{ TOTAL } \\
\cline { 2 - 4 } & $\mathbf{1}^{\mathrm{a}}$. & $\mathbf{2}^{\mathbf{a}}$. & $\mathbf{3}^{\mathbf{a}}{ }^{*}$ & \\
\hline Ext. de Eucalipto & $0,75 \mathrm{~b}$ & 50,00 & 102,75 & $153,50 \mathrm{~b}$ \\
\hline Ext. Cola Caballo & $1,75 \mathrm{~b}$ & 70,00 & 90,00 & $161,75 \mathrm{~b} *$ \\
\hline Ext. Ortiga & $1,25 \mathrm{~b}$ & 90,25 & 89,50 & $181,00 \mathrm{ab}$ \\
\hline Ext. Manzanilla & $27,00 \mathrm{a}$ & 98,50 & 69,25 & $194,75 \mathrm{ab}$ \\
\hline Ext. Caléndula & $1,75 \mathrm{~b}$ & 73,25 & 91,50 & $166,5 \mathrm{~b}$ \\
\hline Ext. Yerbabuena & $7,25 \mathrm{~b}$ & 81,50 & 75,75 & $164,5 \mathrm{~b}$ \\
\hline Ext. Ajo & $13,25 \mathrm{ab}$ & 87,50 & 75,24 & $175,99 \mathrm{~b}$ \\
\hline Ext. Clavo & $3,75 \mathrm{~b}$ & 69,25 & 111,00 & $175,99 \mathrm{~b}$ \\
\hline Ridomil & $14,00 \mathrm{ab}$ & 85,25 & 72,75 & $172,00 \mathrm{~b}$ \\
\hline Testigo & $2,71 \mathrm{~b}$ & 75,75 & 100,00 & $178,46 \mathrm{~b}$ \\
\hline
\end{tabular}

Letras diferentes presentan diferencias estadísticamente significativas $(p<0.05)$

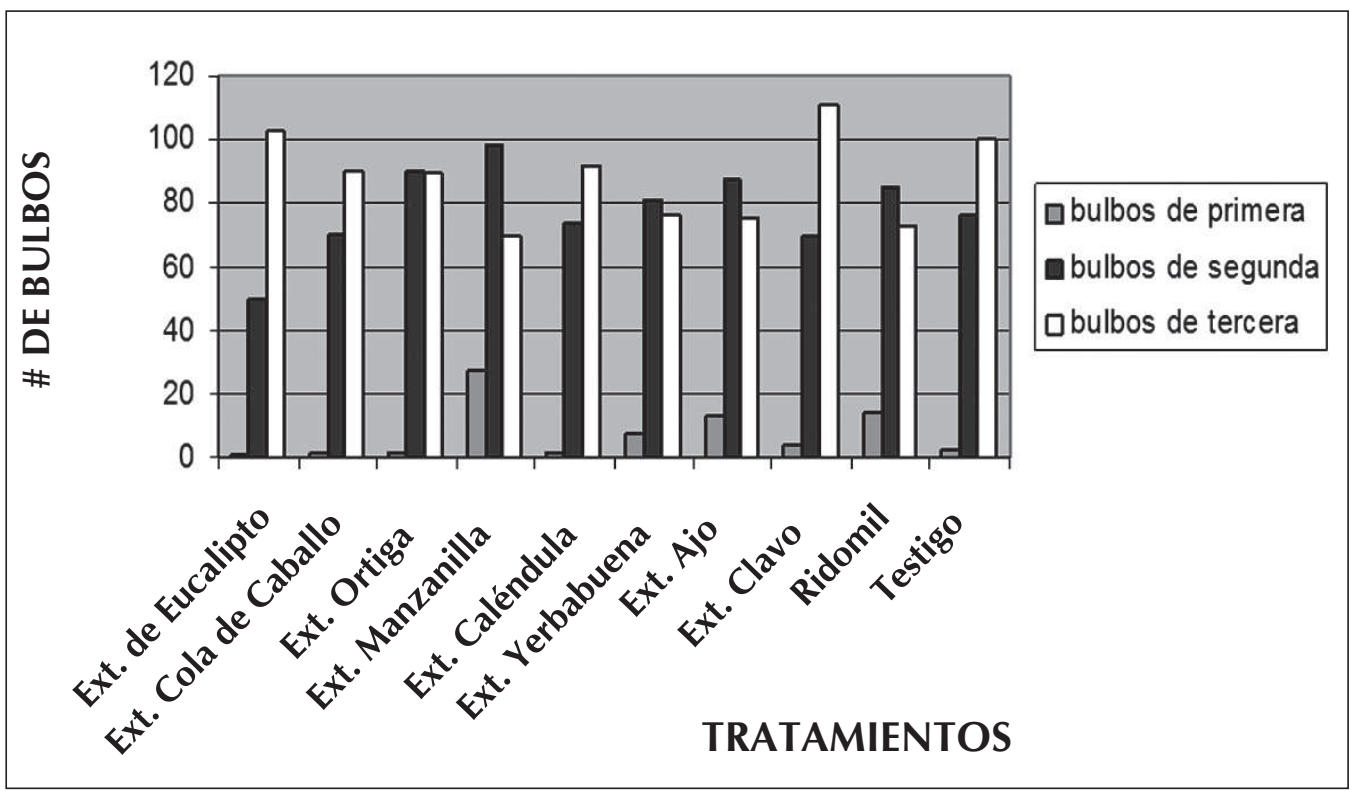

Figura 3. Número de bulbos cosechados con la aplicación de los extractos

Peso de los bulbos. El ANOVA mostró que con la aplicación de los extractos vegetales se obtuvieron diferencias estadísticas significativas en el peso de bulbos de primera, y no en el de los bulbos de segunda, tercera y en el total de los bulbos

El extracto de manzanilla mostró un mejor peso en los bulbos de primera $(3762,5 \mathrm{~g})$, superando con diferencias estadísticas el obtenido con los demás extractos. El extracto de manzanilla superó a los extractos de caléndula y ajo, y al testigo químico en el peso de los bulbos de primera sin diferencias estadísticas entre sí. Para los pesos de los bulbos cosechados de segunda y tercera no se observó ninguna diferencia entre los tratamientos.

Evaluación de extractos de ocho especies vegetales en el control de mildeo velloso (Peronospora destructor Berk) en cebolla de bulbo (Allium cepa L.). - 87 
Tabla 3. Peso de los bulbos cosechados con la aplicación de los tratamientos según su peso

\begin{tabular}{|c|c|c|c|c|c|}
\hline \multirow{2}{*}{ TRATAMIENTO } & \multicolumn{2}{|c|}{ PESO DE BULBOS/GRAMOS } & \multirow{2}{*}{ TOTAL } & $\begin{array}{c}\text { INCREMENTO } \\
\text { PESO (\%) }\end{array}$ \\
\cline { 2 - 5 } & $\mathbf{1 .}^{\mathbf{a}}$ & $\mathbf{2 .}^{\mathbf{a}}$ & $\mathbf{3 .}^{\mathbf{a}}$ & & 83,9 \\
\hline Ext. Eucalipto & $125,000 \mathrm{~b}$ & 3387,500 & 2925,000 & $6437,5 \mathrm{~b}^{*}$ & 94,7 \\
\hline Ext. Cola Caballo & $293,750 \mathrm{~b}$ & 4025,000 & 2950,000 & $7268,75 \mathrm{~b}$ & 120,3 \\
\hline Ext. Ortiga & $200,000 \mathrm{~b}$ & 6155,000 & 2881,250 & $9236,25 \mathrm{ab}$ & 186,2 \\
\hline Ext. Caléndula & $306,250 \mathrm{~b}$ & 5200,000 & 2668,750 & $8175 \mathrm{ab}$ & 106,5 \\
\hline Ext. Yerbabuena & $1097,500 \mathrm{~b}$ & 5100,000 & 2350,000 & $8547,5 \mathrm{ab}$ & 111,4 \\
\hline Ext. Ajo & $2100,00 \mathrm{ab}$ & 6825,000 & 2725,000 & $11.650,0 \mathrm{a}$ & 151,8 \\
\hline Ext. Clavo & $675,000 \mathrm{~b}$ & 4681,250 & 3943,750 & $9300 \mathrm{ab}$ & 121,2 \\
\hline Ridomil & $1950,00 \mathrm{ab}$ & 6375,000 & 2156,250 & $10.481,2 \mathrm{ab}$ & 136,6 \\
\hline Testigo & $526,250 \mathrm{~b}$ & 4456,25 & 2690,000 & $7672.5 \mathrm{~b}$ & 100 \\
\hline
\end{tabular}

Letras diferentes presentan diferencias estadísticamente significativas $(p<0.05)$

El extracto de manzanilla presentó mayor peso total de bulbos cosechados, superando al extracto de eucalipto. Los demás tratamientos no presentaron diferencias estadísticas significativas. Los peores promedios de peso total lo obtuvieron los extractos de cola de caballo y de eucalipto.

En el peso total de bulbos cosechados, el testigo absoluto no presentó diferencias estadísticas del $5 \%$ con el testigo químico Ridomil y con el extracto de manzanilla.
El extracto de eucalipto presentó el menor peso total de bulbos, superando al testigo absoluto y al extracto de ajo, que presentó el segundo mejor promedio de peso total de bulbos.

En el caso de los extractos de eucalipto (eucaliptol) y de cola de caballo (ácido salicílico y aconítico) el incremento en el peso total de los bulbos fue menor al registrado con el testigo absoluto, posiblemente debido a algún efecto fitotóxico, que fue registrado por Rojas (2001).

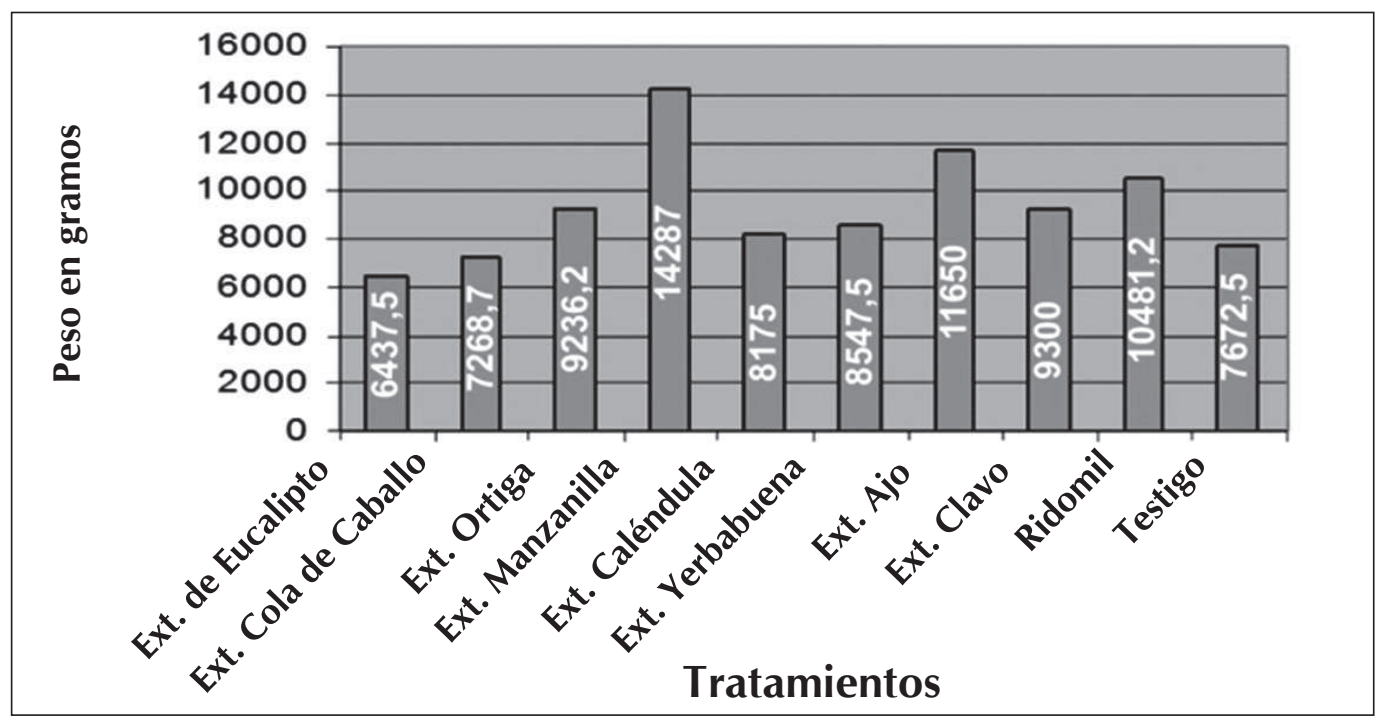

Figura 5. Peso total de los bulbos 


\section{Literatura citada}

Agrios, G. N. (1996). Patología vegetal. México: Limusa.

Alcaldía Municipal de Duitama. Información geográfica del municipio de Duitama. [en línea]. 2012. URL Disponible en: < http://www.duitama-boyaca.gov.co/ nuestromunicipio.shtml?apc $=\mathrm{ml} x \mathrm{x}-1$ $\& m=f>$. [Fecha de acceso 20 de enero de 2012].

Aponte, L. y Alfonso, M. (2008). Cultivos protegidos con técnica hidropónica y biológica. Tomo II. Bogotá: Grupo Técnico Aponte.

Brewster, J. L. (2001). Las cebollas y otros Alliums: Clasificación, orígenes, distribución e importancia económica de los cultivos más importantes. España: Acribia S.A.

Espinel, C. y Neira, A. (1998). Evaluación del extracto de diez especies de plantas medicinales en el control de Colletotrichum en arveja Pisum sativum en condiciones de laboratorio. Universidad Pedagógica y Tecnológica de Colombia.

Gayón, M. E. (2002). Evaluación del extracto de once especies de plantas medicinales en el control de Monilia fructigena en durazno en condiciones de laboratorio. Tesis de Ing. Agronómica, Universidad Pedagógica y Tecnológica de Colombia.
Hard, M. y Araya, E. (1996). Evaluación de fungicidas para el combate del Mildeo velloso de la vid en Costa Rica. Universidad de Costa Rica. X Congreso Nacional Agronómico. III Congreso de Fitopatología.

Rojas, J. (2001). Evaluación de seis especies de plantas con diferentes formas de preparación en el control de Sclerotium cepivorum en cebolla. Tesis de ingeniería agronómica, Universidad Pedagógica y Tecnológica de Colombia.

Rubio, N. y Tobar, F. (2009). Evaluación del efecto de aplicación alternada con el fungicida Ridomil en el control de mildeo velloso en el cultivo de cebolla. Tesis de ingeniería agronómica, Universidad Pedagógica y Tecnológica de Colombia.

Rodríguez, J. y Velandia, H. (1996). Evaluación del extracto de 10 especies de plantas medicinales por su eficacia para el control de la Antracnosis de la curuba en condiciones de laboratorio. Tesis ingeniería agronomía, Universidad Pedagógica y Tecnológica de Colombia.

Fecha de Recepción: 16 de marzo de 2012

Fecha de Aceptación: 10 de abril de 2013 